\title{
Changes in the gene expression programs of renal mesangial cells during diabetic nephropathy
}

\author{
Eric W Brunskill and S Steven Potter ${ }^{*}$
}

\begin{abstract}
Background: Diabetic nephropathy is the leading cause of end stage renal disease. All three cell types of the glomerulus, podocytes, endothelial cells and mesangial cells, play important roles in diabetic nephropathy. In this report we used Meis1-GFP transgenic mice to purify mesangial cells from normal mice and from $\mathrm{db} / \mathrm{db}$ mice, which suffer diabetic nephropathy. The purpose of the study is to better define the unique character of normal mesangial cells, and to characterize their pathogenic and protective responses during diabetic nephropathy.
\end{abstract}

Methods: Comprehensive gene expression states of the normal and diseased mesangial cells were defined with microarrays. By comparing the gene expression profiles of mesangial cells with those of multiple other renal cell types, including podocytes, endothelial cells and renal vesicles, it was possible to better define their exceptional nature, which includes smooth muscle, phagocytic and neuronal traits.

Results: The complete set of mesangial cell expressed transcription factors, growth factors and receptors were identified. In addition, the analysis of the mesangial cells from diabetic nephropathy mice characterized their changes in gene expression. Molecular functions and biological processes specific to diseased mesangial cells were characterized, identifying genes involved in extracellular matrix, cell division, vasculogenesis, and growth factor modulation. Selected gene changes considered of particular importance to the disease process were validated and localized within the glomuerulus by immunostaining. For example, thrombospondin, a key mediator of TGF $\beta$ signaling, was upregulated in the diabetic nephropathy mesangial cells, likely contributing to fibrosis. On the other hand the decorin gene was also upregulated, and expression of this gene has been strongly implicated in the reduction of TGF $\beta$ induced fibrosis.

Conclusions: The results provide an important complement to previous studies examining mesangial cells grown in culture. The remarkable qualities of the mesangial cell are more fully defined in both the normal and diabetic nephropathy diseased state. New gene expression changes and biological pathways are discovered, yielding a deeper understanding of the diabetic nephropathy pathogenic process, and identifying candidate targets for the development of novel therapies.

Keywords: Mesangial cells, Diabetic nephropathy, Fibrosis

\footnotetext{
* Correspondence: steve.potter@cchmc.org

Division of Developmental Biology, Children's Hospital Medical Center,

Cincinnati, Ohio 45229, USA
} 


\section{Background}

The glomerulus is composed of three main cell types, podocytes, endothelial cells and mesangial cells. Mesangial cells provide the extracellular matrix framework upon which the glomerulus is built. In addition they have smooth muscle-like properties and are able to regulate capillary flow. Mesangial cells also have a phagocytic function, and are capable of carrying out disposal and degradation of filtrate debris.

Mesangial cells play important roles in many kidney diseases, including diabetic nephropathy. Type2 diabetes is showing increasing global prevalence and is now the most common cause of end stage renal disease [1]. All three major cell types of the glomerulus have been implicated in diabetic nephropathy. The mesangial cells are responsible for the observed accumulation of extracellular matrix and mesangial expansion [2].

Because of their remarkable properties mesangial cells have been the subject of intense investigation. In particular, many studies have used microarrays to better understand these cells. In most of these studies mesangial cells were grown in culture and exposed to treatments relevant to various disease conditions, and the resulting changes in gene expression profiles were determined with microarrays. Treatments included high glucose [3], static pressure [4], serum [5], kynurenine metabolites [6], endothelin [7] and PDGF [8]. The results defined the multiple gene expression responses of mesangial cells to these stimuli, and thereby provided insight into the molecular mechanisms of mesangial cell mediated pathogenesis. These studies were limited, however, by the use of mesangial cells in culture, which cannot fully replicate the in vivo cell, and by the restricted sets of treatments, which cannot completely reproduce the complex micro-environmental changes experienced by mesangial cells during diabetic nephropathy.

In this report we define in vivo mesangial cell gene expression programs during health and disease. The Meis1-GFP transgene specifically marks mesangial cells within the glomerulus, allowing their purification through fluorescence activated cell sorting (FACS), and subsequent gene expression profiling with microarrays. By comparing the mesangial gene expression pattern to those of other renal cell types it was possible to begin to define the unique character of these remarkable cells. In addition we purified mesangial cells from $d b / d b$ mutant mice with diabetic nephropathy, allowing the determination of both pathogenic and protective changes in gene expression as a function of disease.

\section{Methods}

Purification of mesangial cells

Meis1-GFP transgenic mice, $\operatorname{Tg}($ Meis1-EGFP)FO156Gsat, were obtained from GENSAT/MMRC (http://www.
gensat.org/MMRRC_report.jsp). The Meis1-GFP marks both stromal (interstitial) cells and mesangial cells, so it was essential to first purify glomeruli, to remove stroma. Glomeruli were isolated by sieving, a single cell suspension made, and mesangial cells FACS sorted as previously described [9]. The $d b / d b$ Meis1-GFP mice were made by crossing $d b /+$ and $d b /+$ Meis1-GFP mice, with one eighth of progeny giving the desired genotype.

This study was carried out in strict accordance with the recommendations of the Guide for the Care and Use of Laboratory Animals of the National Institutes of Health. The protocol was approved by the Cincinnati Children's Research Foundation Institutional Animal Care and Use Committee (protocol number 0D02013).

\section{Purity}

The microarray data was analyzed to insure the purity of the mesangial cells. As described in Results we screened for podocyte specific genes and found several that showed low, near background, expression in the mesangial cell profiles, arguing for very limited podocyte contamination. A similar analysis of genes associated with endothelial cells was performed giving similar results. The endothelial specific markers Pecam1, KDR, Tspan8, Plac8 and Eltd1 all showed strong expression in endothelial cells, as expected, and very low expression in the mesangial cells. It is interesting to note, however, that some endothelial specific marker genes did show significant expression in the mesangial cells. For example Tek, also known as Tie2, showed expression levels of about 3,000 in the glomerular endothelial cells, but also a considerable expression level of about 1,000 in mesangial cells. We argue that this is not the result of contamination since other endothelial cell markers showed low expression in the mesangial cells, but rather reflected a partial endothelial phenotype for the mesangial cells. We also examined the expression data for the presence of marker genes from other compartments of the kidney. For example Ace, Acsm1, Aqp11, Slc18a1, Slc22a6 and Spp 2 all should show restricted expression in the proximal tubule of the kidney [10], and each showed very low expression levels in the mesangial cells.

\section{RNA purification and Target Amplification}

RNA was purified using Qiagen RNeasy Micro Kits. Target amplifications were carried out using RiboSpia technology from Nugen, with the Ovation Pico WTA system. Affymetrix standard methods were used for carrying out microarray hybridizations, washes and scans.

\section{Array data analysis}

Data was analyzed primarily using Agilent GeneSpring 11.5.1 software. Specific parameters are described in Results. In addition we used ToppGene (http://toppgene. 
cchmc.org/) [11], ToppCluster (http://toppcluster.cchmc. org/) [12] and Cytoscape (http://www.cytoscape.org/) [13] for functional analysis and preparation of figures.

Microarray data is available on the public resource GUDMAP (www.gudmap.org).

\section{Results and discussion}

The unique gene expression character of the mesangial cell

Mice with the BAC Meis1-GFP transgene show specific GFP labeling of both stromal and mesangial cells in the kidney [10]. It was therefore possible to isolate a very pure population of mesangial cells from adult mouse kidneys by first purifying the glomeruli, using a sieving procedure, followed by preparation of a single cell suspension and FACS sorting of the GFP positive mesangial cells. Samples were prepared in biological triplicate, and gene expression profiles defined using Affymetrix Mouse Gene 1.0 ST microarrays.

To better understand the unique character of mesangial cells we contrasted their gene expression patterns to those of other cell types, including podocytes, endothelial cells, kidney capsule, total kidney cortex, renal vesicles, and cap mesenchyme. The end result was a list of 172 genes with distinctively elevated expression in mesangial cells (Additional file 1: Table S1). A heat map showing the relative expression levels of these genes is shown in Figure 1. (See Additional file 2: Figure S1 for an expandable heat map with genes named). We used the human protein atlas (http://www.proteinatlas.org/) to confirm expression of twenty eight genes predicted by the microarray data to have elevated mesangial cell expression (Figure 2).

To begin to determine the molecular functions and biological processes associated with these genes we used ToppGene (http://toppgene.cchmc.org/). Some of the strongest statistical hits for biological processes, with P-values of essentially zero, were found for wound healing, coagulation, locomotion, regulation of body fluid levels, and muscle contraction. Other biological processes of interest included inflammatory processes, ossification, cell adhesion, and extracellular matrix organization. Quite striking, there was also a gene expression signature of neural character, including behavior, cognition, learning, axogenesis, and neuron development. The picture that emerges describes a remarkable cell type with a markedly diverse set of biological functions. For complete lists see Additional file 3: Table S2.

The results can be presented diagrammatically as cytoscapes (Figure 3). The center hexagons represent some of the genes with mesangial elevated expression, and the surrounding squares are color coded to show molecular functions, biological processes, transcription factors and microRNAs strongly associated with the connected genes.

\section{Transcription factors}

Transcription factors play a key role in determining cell identity. The screen identified fourteen transcription factor genes with significantly elevated expression in mesangial cells, including Gata3, Mecom, Pawr, Arid5b, Zeb2, Ets1, Apbb2, Snai2, Klf12, Hopx, Hdac9, Nr2f1, $E b f 1$ and $R y b p$, as ranked according to expression level.

It has been previously shown that Gata3 is selectively expressed in the ureteric bud as well as mesangial cells and their adjacent endothelial cells during kidney development. GATA3 haploinsufficiency in humans causes hypoparathryoidism, sensineural deafness and renal anomalies, including aplasia, dysplasia and hypoplasia [14].

Mecom, also known as Evi1, encodes a zinc finger transcription factor. It is intriguing to note that MECOM has been shown to physically interact with GATA protein, and it has also been shown to inhibit induction of some TGF $\beta$ target genes, and to play roles in cell proliferation and apoptosis [15].

The PAWR leucine zipper protein was shown to sensitize cells to apoptotic signals and was originally identified as a gene induced in apoptotic prostate cancer cells [16]. Of interest, given mesangial cell function in the synthesis of extracellular matrix, PAWR has also been shown to regulate secretase cleavage of amyloid precursor protein, and has thereby been implicated in Alzheimer disease [17]. PAWR was also shown to interact with WT1 and to enhance its transcriptional repression activity [18]. This might seem irrelevant since Wt1 is generally though to be associated with podocytes, and not mesangial cells. We observed very robust expression of Pawr in mesangial cells, and about a three fold lower level in podocytes. Of considerable interest, however, we also observed an inverse gene expression pattern for $W t 1$, with raw signal expression in podocytes of about 3400 , and a lower but still strong expression level of about 650 in mesangial cells.

This surprisingly suggests that Wt1 is expressed in mesangial cells as well as podocytes. Could this be due to contamination of the mesangial cells with podocytes? We tested the data for possible contamination by examining the mesangial gene expression profile for other genes thought to be podocyte specific. If they all showed significant expression in the putative mesangial cells then this would argue strongly in favor of contamination. If, on the other hand, some are indeed truly podocyte specific in the dataset then this would rule out contamination as an explanation. It is difficult to envisage a scenario where the transcripts of some podocyte 


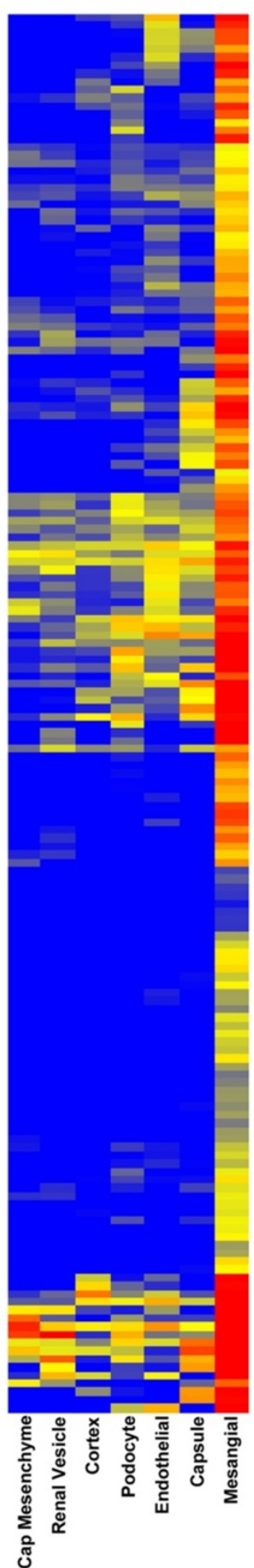

Figure 1 Heatmap showing genes with elevated expression in normal mesangial cells. Expression patterns of 172 genes with up-regulation in mesangial cells are compared across multiple renal compartments, including cap mesenchyme, renal vesicle, total renal cortex, podocytes, endothelial cells and renal capsule. Red indicates high, yellow represents intermediate and blue indicates low gene expression level. Most of the 172 genes are not uniquely expressed in mesangial cells, but do show significantly elevated mesangial expression. For an image that can be zoomed to visualize gene names see Additional file 2: Figure S1.

genes contaminated the purified mesangial cells while others did not.

Further analysis of the data indicated that contamination could not explain the observed Wt1 expression in mesangial cells. Several genes, including Podxl, Nphs1 and Nphs 2 showed strongly podocyte specific expression, as expected, and very low expression levels in mesangial cells. For example, Nphs 2 gave raw signal levels of approximately 5,000 in podocytes, but only about 100 in mesangial cells.

The APBB2 protein, similar to PAWR, was shown to bind to amyloid precursor protein (APP)[19]. It is interesting to note that the gene encoding APP shows strong expression in mesangial cells, with raw signal levels over 4,000 , but is also widely and strongly expressed in the other cell types examined, including podocytes and endothelial cells. $A p b b 2$ has also been shown to be a regulator of programmed cell death [20].

The ARID5B transcription factor has been previously shown to be able to drive smooth muscle development when over expressed in 3T3 fibroblasts [21]. Its strong expression in mesangial cells likely reflects their smooth muscle character. Arid $5 b$ mutant mice show severe reduction in the number of mesangial cells, as well as their mis-location at the edge of the glomerulus [22]. Hорx encodes a divergent homeodomain protein that physically interacts with SRF and regulates gene expression in cardiac muscle [23], and could therefore also play a role in defining the muscle character of mesangial cells.

Zeb2 encodes a 2-handed zinc finger/homeodomain DNA binding transcriptional repressor that interacts with activated SMADs and represses cadherin expression in response to TGF $\beta$ signaling [24]. This is of particular interest since TGF $\beta$ is thought to play an essential role in driving kidney fibrosis during a number of diseases, including diabetic nephropathy. Decreased cadherin expression promotes EMT, the production of fibroblasts, and consequent fibrosis. The Snai2 gene, a member of the snail family, which encodes transcriptional repressors. Notch signaling can activate Snai2, promoting resistance to anoikis, and, once again, inducing epithelia to mesenchyme transition through repression of E-cadherin [25]. 


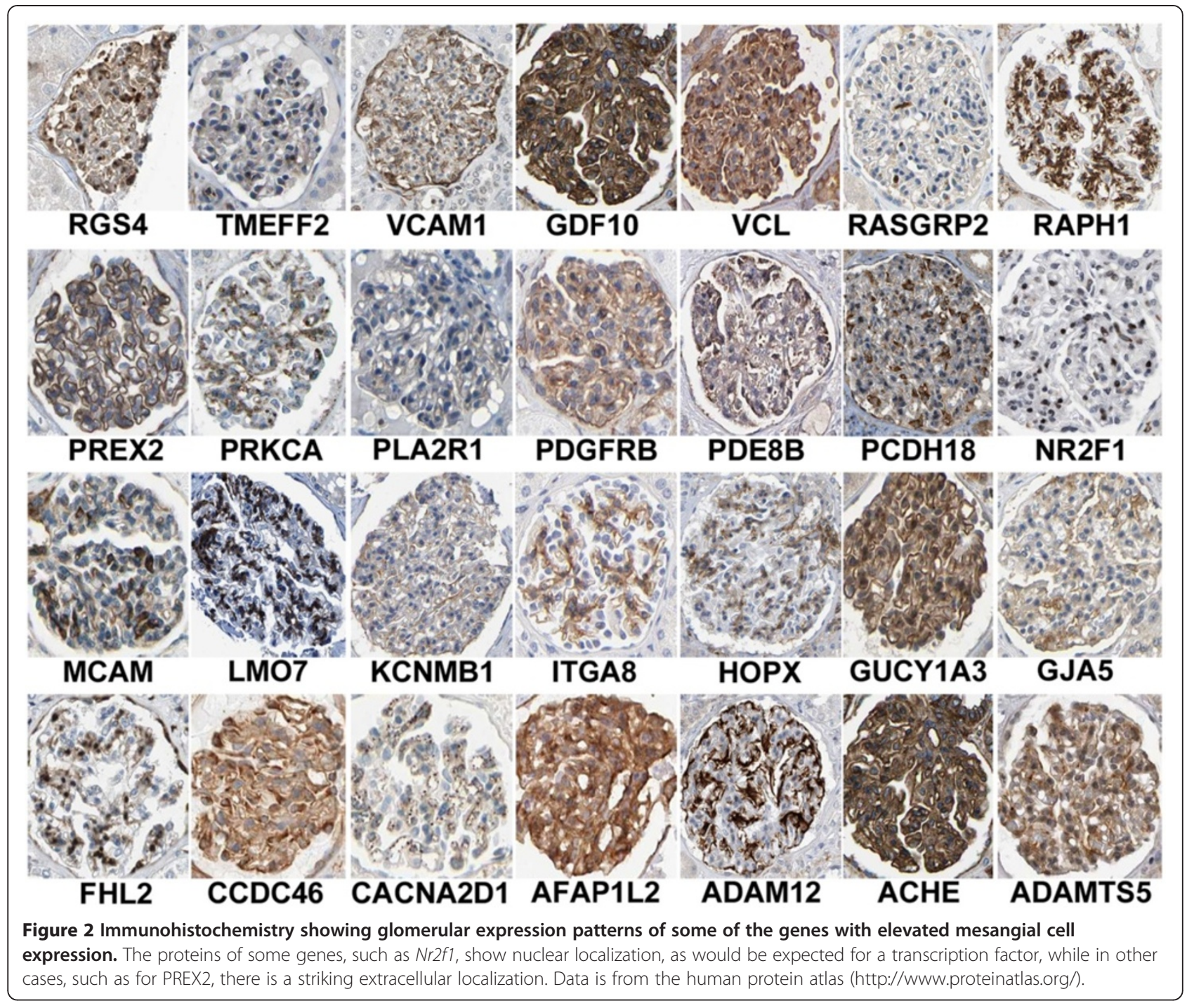

Ets1 is most strongly expressed in lymphoid, endothelial and neural crest cells. It plays a key role in cytokine signaling [26]. Its expression in mesangial cells reflects a gene expression signature with significant endothelial character.

The nuclear receptor Nr2f1 is of interest, as it has been previously shown to have restricted expression in the mesangial cells of the adult kidney, again providing historic validation of the microarray screen.

\section{Growth factors}

Mesangial cells showed very robust expression of three growth factor encoding genes, Ptn, Mfges and Gdf10. The Ptn gene, encoding pleiotrophin, was expressed at a very high level in mesangial cells, with raw signal values of around 6000. Pleiotrophin is a heparin-binding growth/differentiation inducing cytokine and is a powerful angiogenic factor.
PTN receptors include PTPRB, PTRZ, SDC1 and SDC3. In the prostate PTN has been implicated in the autocrine regulation of mesenchyme and the paracrine regulation of epithelia [27]. We observed modest expression levels of two of these receptor genes (Ptprb and $S d c 3$ ) in mesangial cells, suggesting a possible autocrine role. In addition there was extremely strong expression of Ptprb in the glomerular endothelial cells, strongly suggesting a paracrine function. It is also interesting to note that PTN has been shown to disrupt calcium dependent cell-cell adhesion and to promote epithelia to mesenchyme transition [28]. Finally, it has also been observed that PTN has strong antibacterial activity when tested against a panel of gram-positive and gramnegative bacteria [29], arguing that PTN is part of the innate immune system.

Mfge8 (SED1) has many functions, including promoting the phagocytic removal of apoptotic cells [30]. Pericytes were previously identified as a major source of 


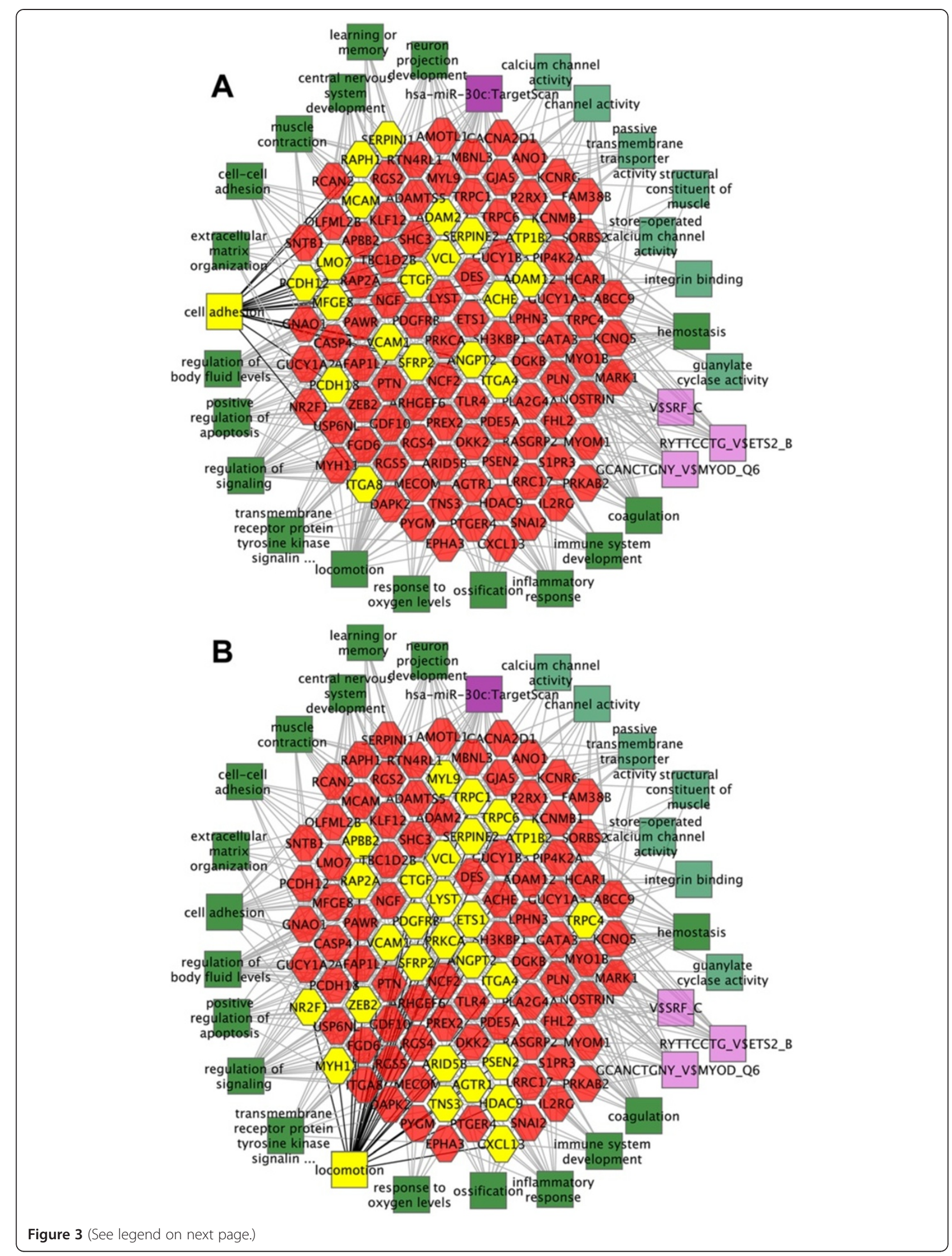


(See figure on previous page.)

Figure 3 Molecular function, biological processes and candidate regulatory transcription factors and microRNAs in normal mesangial cells. The central hexagons show some of the 172 genes with elevated expression in normal mesangial cells, compared to other cell types. The surrounding squares show color coded molecular functions (light green), biological processes (dark green), transcription factors (light purple) and microRNAs (dark purple), strongly associated with these genes. Lines show connections to linked genes. $\mathbf{A}$. The cell adhesion biological process, with associated genes, is highlighted in yellow. B. The locomotion biological process and associated genes is highlighted in yellow. For complete lists of molecular functions, biological processes, transcription factors, microRNAs and other features see Additional file 3: Table S2.

Mfges [31], and of course mesangial cells can be considered specialized pericytes. In Mfges knockout mice tumor and retinopathy associated angiogenesis was reduced [31]. It is also interesting to note that $M f g e 8$ has been shown to potentiate PDGFRB signaling [32].

The final robustly expressed growth factor gene with mesangial cell elevated expression was Gdf10, which is a member of the $\operatorname{Tg} f-\beta$ superfamily and is closely related to Bmp3.

\section{Receptors}

The screen of the microarray data revealed a large number of receptor encoding genes with elevated mesangial expression. The top dozen, ranked according to expression levels, were Itga8, Agtr1a, Pdgfrb, Itga4, S1pr3, Trpc1, Il2rg, Ptger4, Pla2r1, Lphn3, P2rx1 and Tlr4.

Itga8, (integrin alpha8) has been previously shown to have glomerular expression that is restricted to the mesangial cells, providing another historical validation of the microarray dataset. The Agtrla gene encodes a receptor for angiotensin, a potent vasopressor hormone. Agtrla mutant mice show hypertrophy of the juxtaglomerular apparatus and mild mesangial expansion [33].

Pdgfrb has previously been shown to be expressed in mesangial cells, and the Pdfrb mutant mouse shows a failure of mesangial cell development [34]. It is interesting to note that mesangial cells also express $P d g f c$ at robust levels, suggesting a possible autocrine function. $P d g f c$ did not emerge in the screen for mesangial cell enriched growth factors because it is also expressed in other cell types.

The S1pr3 gene encodes a receptor for sphingosine-1phosphate. It has been shown to be expressed by macrophages and to play a role in their recruitment during inflammation and atherosclerosis [35]. Reports have also linked S1pr3 signaling to fibrosis [36].

The Mrc1 receptor binds the surfaces of pathogenic viruses and bacteria, facilitating their engulfment. It is similar, therefore, in function to the secreted MFGE8, which also promotes phagocytosis. Another receptor associated with a macrophage function is IL2RG, a common subunit for the receptors of a variety of interleukins. In addition the mesangial cells showed a high level of expression of Tlr4, which encodes a member of the Toll-like receptors. These are single transmembrane receptors that have a key role in the innate immune system, and are generally found on immune cells. Tlr4 was previously shown to be expressed by mesangial cells, and to contribute to antibody mediated glomerulonephritis through the production of CXC cytokines [37]. The observed expression of S1pr3, Mrc1, Il2rg and Mfges by mesangial cells serves to better define their anti-bacterial macrophage like character.

Several TRPC receptors were expressed at elevated levels in mesangial cells, including TRPC1, TRPC4 and TRPC6. They belong to the transient receptor potential super family of cation channels that have been implicated in receptor and store-operated calcium entry, and cell death, as well as functioning as sensors for heat, cold, stretch, and osmotic changes.

The P2RX1 receptor functions in mediating synaptic transmission from neurons to smooth muscle, and driving vasoconstriction. The PTERG4 receptor has the opposite effect. It is a receptor for prostaglandin E2 and can induce smooth muscle relaxation.

\section{$d b / d b$ mesangial cells}

We next sought to better define the protective and pathogenic gene expression changes that occur in mesangial cells as a function of diabetic nephropathy. We used the $d b / d b$ mouse model, which has a mutation in the leptin receptor gene and as a result suffers obesity, diabetes and diabetic nephropathy [38]. We generated $d b / d b$ mice that carried the Meis1-GFP transgene.

At the age of seven months the $d b / d b$ mice showed significant proteinuria, with albumin levels of $994 \pm 245$ ( $\mu \mathrm{g} / \mathrm{ml} / 24 \mathrm{hrs})$, compared to $281 \pm 67$ for wild type $(\mathrm{p}=0.0036)$. The $d b / d b$ mice also showed dramatic glycosuria, with glucose levels of 7,580 $\pm 1,009 \mathrm{mg} / \mathrm{dL}$, compared to $78 \pm 6$ for wild type. Hematoxylin and eosin staining showed striking glomerular hypertrophy (Figure 4A,B), and Masson's trichome stain indicated elevated levels of collagen deposition in the glomeruli of $d b / d b$ mice (Figure 4C,D). Further, Periodic acid-Shiff (PAS) staining showed clear mesangial expansion in $d b /$ $d b$ mice (Figure 4E,F).

The mesangial cell gene expression data from biological triplicates of wild type and $d b / d b$ mice was analyzed, identifying 184 probesets, with 105 up-regulated and 79 down-regulated in $d b / d b$ mesangial cells (Additional file 4: Table S3). We used Toppgene to perform a functional analysis of the list of genes up-regulated 


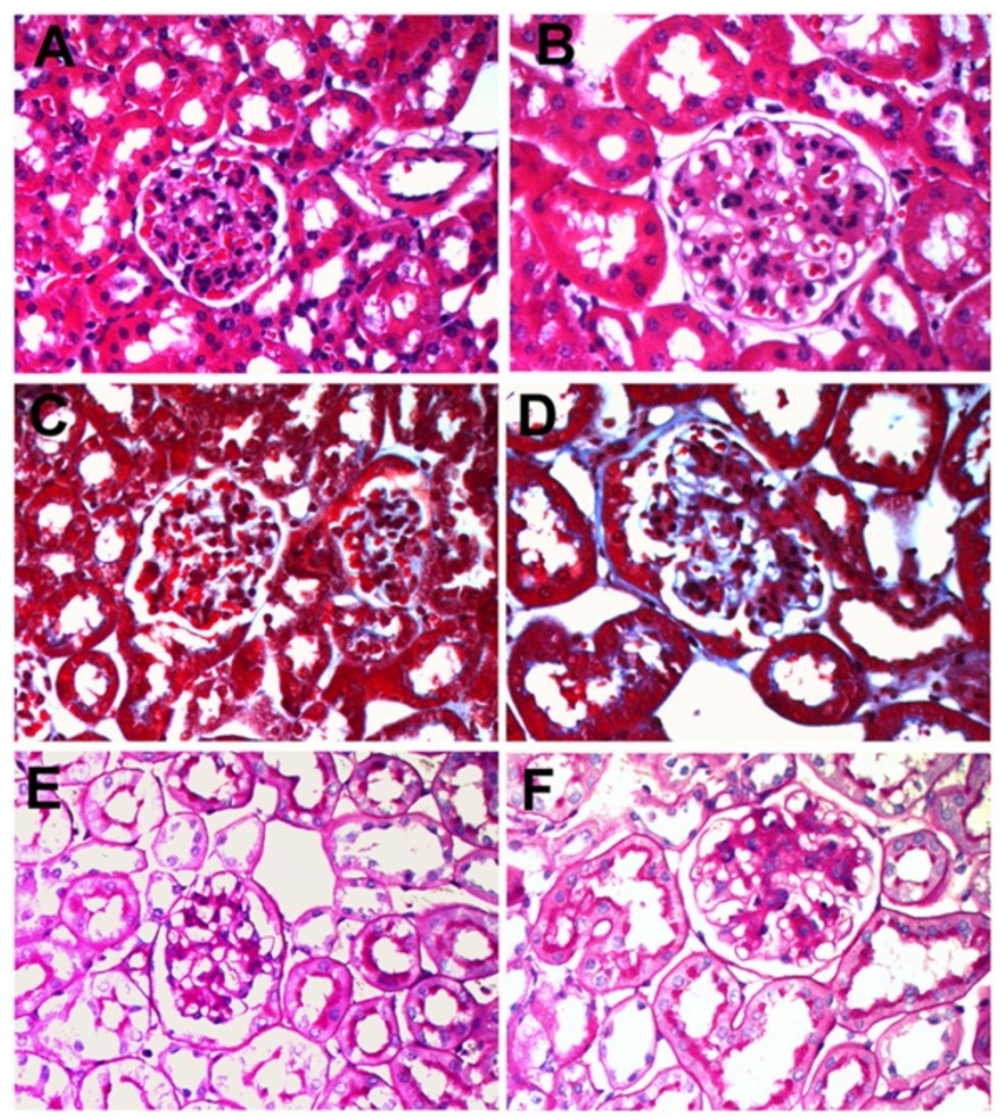

Figure 4 Glomerular changes in $d \boldsymbol{b} / \mathbf{d} b$ diabetic mice. Panels $\mathbf{A}, \mathbf{C}$ and $\mathbf{E}$ are controls, and panels $\mathbf{B}, \mathbf{D}$ and $\mathbf{F}$ are from $d b / d b$ mice with diabetic nephropathy. Panels $\mathbf{A}$ and $\mathbf{B}$ show histological sections stained with hematoxylin and eosin and demonstrate glomeruli hypertrophy. Panels $\mathbf{C}$ and $\mathbf{D}$ are sections stained with Masson's trichrome stain. Note the increased staining (blue) indicating a higher level of collagen deposition in the glomeruli of diabetic mice. Panels $\mathbf{E}$ and $\mathbf{F}$ are sections stained with Periodic acid-Schiff (PAS) and show mesangial expansion in diabetic mice. All images are representative glomeruli at an original magnification of $200 \mathrm{X}$.

(Additional file 5: Table S4). Consistent with the known functions of mesangial cells in the pathogenesis of diabetic nephropathy, the top three molecular functions were extracellular matrix binding, fibronectin binding and laminin binding. Some of the other up-regulated molecular functions of particular interest were Rac GTPase binding, Rho GTPase activator activity, glycoprotein binding, and metallopeptidase activity. The top identified up-regulated biological processes were negative regulation of cell-substrate adhesion, and vascular development. Others included regulation of cell proliferation, which is potentially important in terms of mesangial matrix expansion, immune response, muscle cell differentiation and wound healing. For complete lists see Additional file 5: Table S4. A cytoscape graphics view of some of the up-regulated genes and associated molecular functions, biological processes, and candidate regulating microRNAs is shown in Figure 5.

We then applied a more stringent screen, requiring at least raw signal expression of 300 in three samples, to identify the most robustly expressed up-regulated mesangial cell genes in $d b / d b$ mice. Some of the more interesting of the resulting 34 genes are described below.

Thrombospondin (Tsp1) showed the greatest fold upregulation (2.8 fold change) in mice with diabetic nephropathy. TGF $\beta$ is primarily secreted as an inactive procytokine that must be activated to bind to its receptors. Thrombospondin plays an important role in this activation process, with Tsp1 mutant mice showing an inflammatory phenotype similar to that of $T g f-\beta$ mutant mice $[39,40]$.

TGF $\beta$ has been known for many years to play a major role in renal fibrosis. Indeed, it has been argued that most of the features of diabetic nephropathy, including GBM thickening, matrix expansion, hypertrophy and podocyte loss are the result of TGF $\beta$ signaling [41]. Further, it has been known for some time that mesangial cells produce thrombospondin [42], and high glucose can up-regulate thrombospondin expression in both rat and human mesangial cells [43]. Elevated TSP1 expression in mesangial cells of human type- 2 diabetic nephropathy was previously demonstrated by immunohistochemical 


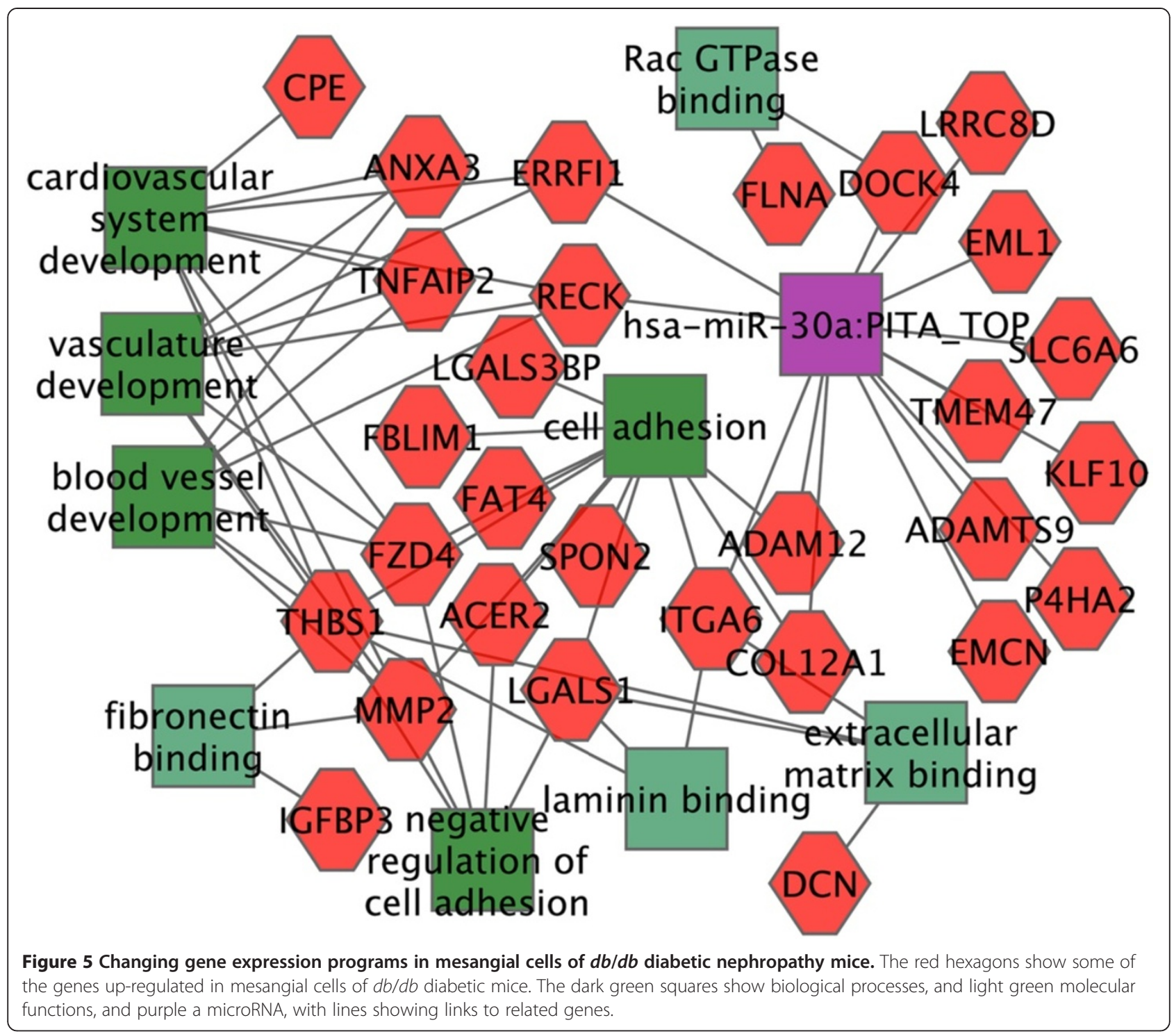

staining of renal biopsies [43]. These results provide additional historic validation of the current study. The gene we identified with the greatest up-regulaton, Tsp1, has already been shown to play an important role in diabetic nephropathy.

The only collagen gene observed to change significantly in expression was Col12a1, which was upregulated almost two fold in $d b / d b$ mice. It is interesting that none of the Col4 subunit genes showed a change in expression, although their upregulation in diabetic nephropathy has been previously reported [44]. This could be reconciled by a decreased rate of collagen degradation in $d b / d b$ glomeruli [45]. There is also evidence that renal fibroblasts, associated with fibrosis, might be an important source of the increased COL4 [46].

Similarly, there was no increased transcription of PKC genes in the $d b / d b$ mesangial cells, although previous reports have documented increased PKC activation as well as total PKC levels in mesangial cells treated with glucose [47]. Again, the likely explanation is a change in PKC turnover.

Emcn, also up-regulated, encodes a mucin like sialoglycoprotein "that interferes with the assembly of focal adhesions complexes and inhibits the interaction between cells and the extracellular matrix" (EMCN Gene Cards) [48]. Other up-regulated genes associated with cell adhesion and the extracellular matrix included Mmp2 (matrix metallopeptidase 2), and Lgals1 (lectin, galactose binding). Other ECM related up-regulated genes, but with raw expression levels below 300, included Fat4, Fblim1, Spon2, Lgals3bp, Adam12, and Itga6.

The annexin ANXA3 was also up regulated in $d b / d b$ mesangial cells. ANXA3 has been linked to phagocytosis 


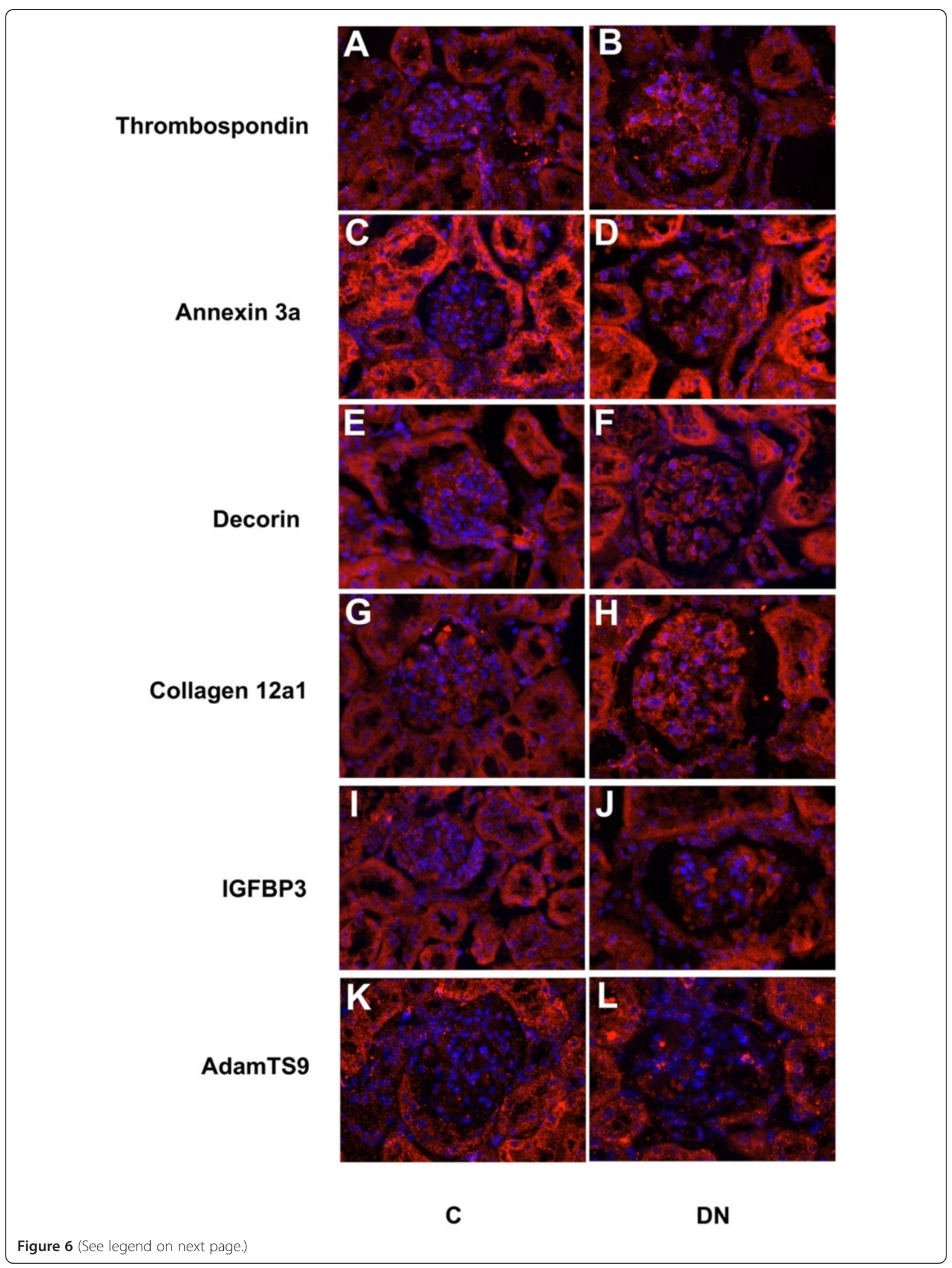


[49], the promotion of angiogenesis [50], the inhibition of phospholipase A2 and anti-coagulation. Ctsl encodes cathepsin L, a lysosomal proteinase that has been shown to play a major role in neovascularization.

We also observed up-regulation of ACER2, a ceramidase that cleaves ceramides to generate sphingosine, which has the opposite effects on cell proliferation as survival as sphingosine-1-phosphate. The up-regulated Ppap $2 a$ gene, encoding phosphatidic acid phosphatase, can dephosphorylate bioactive glycerolipids as well as sphingolipids.

Elevated Acta2 expression, encoding smooth muscle actin, has been noted previously in diabetic nephropathy mesangial cells, and has been associated with differentiation to a myofibroblast phenotype, believed causally related to fibrosis. Of interest, TGF $\beta$ has been shown to induce Acta2 in mesangial cells grown in culture [51]. We also observed up regulation of Flna, encoding filamin A, alpha, which is an actin binding protein involved in cytoskeleletal remodeling to effect changes in cell shape and migration. Another biologically related upregulated gene was $W d r 1$, which induces the disassembly of actin filaments.

Of particular interest, the Decorin gene, Dcn, showed up-regulation in $d b / d b$ mesangial cells. Decorin has been shown to interact with many extracellular matrix molecules, including collagen, fibronectin and thrombospondin, as well as the growth factor TGF $\beta$ [52]. Expression of $D c n$ has been shown to reduce TGF $\beta$ induced fibrosis in many model systems [53]. Indeed, it has been shown that decorin deficiency results in a much more severe diabetic nephropathy in mice with with streptozotocin induced diabetes [54]. This strongly suggests that the over expression of Dcn observed in $d b / d b$ mesangial cells serves a protective function.

\section{Validation}

To confirm the microarray predicted expression changes we performed immunohistochemisty, comparing wild type glomeruli to those of $d b / d b$ mice (Figure 6). The results showed elevated $d b / d b$ glomerular expression of Thrombospondin, Annexin3a, Decorin, Collagen12a1, Igfbp3 and AdamTS9, consistent with the microarray results. As might be expected, there were typically restricted regions of over expression. This facilitated detection, since the overall fold change in expression compared to wild type was generally relatively small. Of interest, these genes also showed expression in flanking tubules, which was generally unchanged in the $d b / d b$ mouse, although increased somewhat for Decorin and Collagen 12a1.

\section{Conclusions}

In conclusion, we have FACS purified mesangial cells from both normal and diabetic nephropathy model mice and defined their in vivo gene expression states. The results contribute to our molecular understanding of the multifunctional character of mesangial cells, with their gene expression signatures showing similarities to pericytes, muscle cells, endothelial cells, neurons, and phagocytes. The expression levels of all growth factor, receptor and transcription factor genes were defined. In addition we analyzed the changes in mesangial gene expression occurring as a function of diabetic nephropathy, identifying genes involved in extracellular matrix, cell division, vasculogenesis, and growth factor modulation. New gene expression changes and biological pathways were discovered, yielding a deeper understanding of the disease process, and candidate targets for the development of novel therapies.

\section{Additional files}

Additional file 1: Table S1. List of 172 genes with elevated expression in normal mesangial cells compared to other renal compartments. Fold change shown is relative to renal vesicles.

Additional file 2: Figure S1. Heatmap showing genes with elevated expression in normal mesangial cells. Expression patterns of 172 genes with up-regulation in mesangial cells are compared across multiple renal compartments, including cap mesenchyme, renal vesicle, total renal cortex, podocytes, endothelial cells and renal capsule. Red indicates high, yellow represents intermediate and blue indicates low gene expression level. This figure can be zoomed to visualize individual gene names.

Additional file 3: Table S2. Functional analysis of genes with elevated expression in normal mesangial cells. The top molecular functions, biological processes, cellular components, evolutionarily conserved transcription factor binding sites, microRNAs and diseases associated with the list of 172 genes showing elevated mesangial cell expression. For example, the first molecular function is store-operated calcium channel activity. There are seven genes associated with this function and three of them (Trpc1, Trpc4 and Trpc6) show elevated expression in mesangial cells, giving an uncorrected P-value of 0.000017 . Functional analysis was carried out with the ToppGene (http://toppgene.cchmc.org/) web tool.

Additional file 4: Table S3. Genes with altered expression in diabetic nephropathy mesangial cells. The left column lists the Affymetrix probeset IDs. For some genes there are multiple probesets. Column B shows fold change, comparing wild type to $d b / d b$ mutant. Column $C$ shows directionality of change, with down signifying lower in wild type. The gene with the greatest fold change was Thrombospondin, which was 2.8 fold down in wild type compared to mutant. 
Additional file 5: Table S4. Functional analysis of genes up-regulated in $d b / d b$ diabetic nephropathy mesangial cells. The top molecular function, biological processes, cellular components, evolutionarily conserved transcription factor binding sites and diseases associated with the genes showing up-regulation in $d b / d b$ mesangial cells, as determined with the ToppGene (http://toppgene.cchmc.org/) web tool.

\section{Competing interests}

The authors declare no conflicts of interest.

\section{Acknowledgements}

We thank Douglas Meyer for technical assistance. This work was supported by grant RO1DK081489 from the $\mathrm{NIH}$.

\section{Authors' contributions}

E.W.B. performed the experiments and generated the data. S.S.P. and E.W.B. analyzed the data. S.S.P. wrote the manuscript and E.W.B. reviewed/edited the manuscript. Both authors read and approved the final manuscript.

Received: 27 May 2012 Accepted: 11 July 2012

Published: 28 July 2012

\section{References}

1. Atkins RC, Zimmet P: Diabetic kidney disease: act now or pay later. Nephrol Dial Transplant 2010, 25(2):331-333.

2. Steffes MW, Bilous RW, Sutherland DE, Mauer SM: Cell and matrix components of the glomerular mesangium in type I diabetes. Diabetes 1992, 41(6):679-684.

3. Kobayashi T, Uehara S, Ikeda T, Itadani H, Kotani H: Vitamin D3 upregulated protein-1 regulates collagen expression in mesangial cells. Kidney Int 2003, 64(5):1632-1642.

4. Hishikawa K, Oemar BS, Nakaki T: Static pressure regulates connective tissue growth factor expression in human mesangial cells. J Biol Chem 2001, 276(20):16797-16803.

5. Mishra R, Leahy P, Simonson MS: Gene expression profiling reveals role for EGF-family ligands in mesangial cell proliferation. Am J Physiol Renal Physiol 2002, 283(5):F1151-F1159.

6. Yoshimura H, Sakai T, Kuwahara Y, Ito M, Tsuritani K, Hirasawa Y, Nagamatsu $\mathrm{T}$ : Effects of kynurenine metabolites on mesangial cell proliferation and gene expression. Exp Mol Pathol 2009, 87(1):70-75

7. Simonson MS, Ismail-Beigi F: Endothelin-1 increases collagen accumulation in renal mesangial cells by stimulating a chemokine and cytokine autocrine signaling loop. J Biol Chem 2011, 286(13):11003-11008.

8. Sadlier DM, Ouyang X, McMahon B, Mu W, Ohashi R, Rodgers K, Murray D, Nakagawa T, Godson C, Doran P, et al: Microarray and bioinformatic detection of novel and established genes expressed in experimental anti-Thy1 nephritis. Kidney Int 2005, 68(6):2542-2561.

9. Brunskill EW, Georgas K, Rumballe B, Little MH, Potter SS: Defining the molecular character of the developing and adult kidney podocyte. PLOS One 2011, 6(9):e24640.

10. Brunskill EW, Aronow BJ, Georgas K, Rumballe B, Valerius MT, Aronow J, Kaimal $V$, Jegga AG, Yu J, Grimmond S, et al: Atlas of gene expression in the developing kidney at microanatomic resolution. Dev Cell 2008, 15 (5):781-791.

11. Chen J, Bardes EE, Aronow BJ, Jegga AG: ToppGene Suite for gene list enrichment analysis and candidate gene prioritization. Nucleic Acids Res 2009, 37(Web Server issue):W305-W311.

12. Kaimal V, Bardes EE, Tabar SC, Jegga AG, Aronow BJ: ToppCluster: a multiple gene list feature analyzer for comparative enrichment clustering and network-based dissection of biological systems. Nucleic Acids Res 2010, 38(Web Server issue):W96-W102.

13. Shannon P, Markiel A, Ozier O, Baliga NS, Wang JT, Ramage D, Amin N, Schwikowski B, Ideker T: Cytoscape: a software environment for integrated models of biomolecular interaction networks. Genome Res 2003, 13(11):2498-2504.

14. Hernandez AM, Villamar M, Rosello L, Moreno-Pelayo MA, Moreno F, Del Castillo I: Novel mutation in the gene encoding the GATA3 transcription factor in a Spanish familial case of hypoparathyroidism, deafness, and renal dysplasia (HDR) syndrome with female genital tract malformations. Am J Med Genet A 2007, 143(7):757-762.

15. Wieser R: The oncogene and developmental regulator EVI1: expression, biochemical properties, and biological functions. Gene 2007, 396(2):346357.

16. Gurumurthy S, Rangnekar VM: Par-4 inducible apoptosis in prostate cancer cells. J Cell Biochem 2004, 91(3):504-512.

17. Xie J, Guo Q: PAR-4 is involved in regulation of beta-secretase cleavage of the Alzheimer amyloid precursor protein. J Biol Chem 2005, 280 (14):13824-13832.

18. Johnstone RW, See RH, Sells SF, Wang J, Muthukkumar S, Englert C, Haber DA, Licht JD, Sugrue SP, Roberts T, et al: A novel repressor, par-4, modulates transcription and growth suppression functions of the Wilms' tumor suppressor WT1. Mol Cell Biol 1996, 16(12):6945-6956.

19. Guenette SY, Chen J, Jondro PD, Tanzi RE: Association of a novel human FE65-like protein with the cytoplasmic domain of the beta-amyloid precursor protein. Proc Natl Acad Sci U S A 1996, 93(20):10832-10837.

20. Lange A, Thon L, Mathieu S, Adam D: The apoptosis inhibitory domain of FE65-like protein 1 regulates both apoptotic and caspase-independent programmed cell death mediated by tumor necrosis factor. Biochem Biophys Res Commun 2005, 335(2):575-583.

21. Watanabe M, Layne MD, Hsieh CM, Maemura K, Gray S, Lee ME, Jain MK: Regulation of smooth muscle cell differentiation by AT-rich interaction domain transcription factors Mrf2alpha and Mrf2beta. Circ Res 2002, 91 (5):382-389.

22. Schmahl J, Raymond CS, Soriano P: PDGF signaling specificity is mediated through multiple immediate early genes. Nat Genet 2007, 39(1):52-60

23. Chen F, Kook H, Milewski R, Gitler AD, Lu MM, Li J, Nazarian R, Schnepp R, Jen K, Biben C, et al: Hop is an unusual homeobox gene that modulates cardiac development. Cell 2002, 110(6):713-723.

24. Wang B, Herman-Edelstein M, Koh P, Burns W, Jandeleit-Dahm K, Watson A, Saleem M, Goodall GJ, Twigg SM, Cooper ME, et al: E-cadherin expression is regulated by miR-192/215 by a mechanism that is independent of the profibrotic effects of transforming growth factor-beta. Diabetes 2010, 59 (7):1794-1802.

25. Leong KG, Niessen K, Kulic I, Raouf A, Eaves C, Pollet I, Karsan A: Jagged1mediated Notch activation induces epithelial-to-mesenchymal transition through Slug-induced repression of E-cadherin. J Exp Med 2007, 204 (12):2935-2948.

26. Russell L, Garrett-Sinha LA: Transcription factor Ets-1 in cytokine and chemokine gene regulation. Cytokine 2010, 51(3):217-226.

27. Orr B, Vanpoucke G, Grace OC, Smith L, Anderson RA, Riddick AC, Franco OE, Hayward SW, Thomson AA: Expression of pleiotrophin in the prostate is androgen regulated and it functions as an autocrine regulator of mesenchyme and cancer associated fibroblasts and as a paracrine regulator of epithelia. Prostate 2011, 71(3):305-317.

28. Perez-Pinera P, Alcantara S, Dimitrov T, Vega JA, Deuel TF: Pleiotrophin disrupts calcium-dependent homophilic cell-cell adhesion and initiates an epithelial-mesenchymal transition. Proc Natl Acad Sci U S A 2006, 103 (47):17795-17800.

29. Svensson SL, Pasupuleti M, Walse B, Malmsten M, Morgelin M, Sjogren C, Olin Al, Collin M, Schmidtchen A, Palmer R, et al: Midkine and pleiotrophin have bactericidal properties: preserved antibacterial activity in a family of heparin-binding growth factors during evolution. J Biol Chem 2010, 285(21):16105-16115.

30. Raymond A, Ensslin MA, Shur BD: SED1/MFG-E8: a bi-motif protein that orchestrates diverse cellular interactions. J Cell Biochem 2009, 106(6): 957-966.

31. Motegi S, Leitner WW, Lu M, Tada Y, Sardy M, Wu C, Chavakis T, Udey MC: Pericyte-derived MFG-E8 regulates pathologic angiogenesis. Arterioscler Thromb Vasc Biol 2011, 31(9):2024-2034.

32. Motegi S, Garfield S, Feng X, Sardy M, Udey MC: Potentiation of plateletderived growth factor receptor-beta signaling mediated by integrinassociated MFG-E8. Arterioscler Thromb Vasc Biol 2011, 31(11):2653-2664

33. Oliverio MI, Madsen K, Best CF, Ito M, Maeda N, Smithies O, Coffman TM: Renal growth and development in mice lacking AT1A receptors for angiotensin II. Am J Physio/ 1998, 274(1 Pt 2):F43-F50.

34. Soriano $\mathrm{P}$ : Abnormal kidney development and hematological disorders in PDGF beta-receptor mutant mice. Genes Dev 1994, 8(16):1888-1896. 
35. Keul P, Lucke S, von Wnuck Lipinski K, Bode C, Graler M, Heusch G, Levkau B: Sphingosine-1-phosphate receptor 3 promotes recruitment of monocyte/macrophages in inflammation and atherosclerosis. Circ Res 2011, 108(3):314-323.

36. Takuwa N, Ohkura S, Takashima S, Ohtani K, Okamoto Y, Tanaka T, Hirano K, Usui S, Wang F, Du W, et al: S1P3-mediated cardiac fibrosis in sphingosine kinase 1 transgenic mice involves reactive oxygen species. Cardiovasc Res 2010, 85(3):484-493.

37. Brown HJ, Lock HR, Wolfs TG, Buurman WA, Sacks SH, Robson MG: Toll-like receptor 4 ligation on intrinsic renal cells contributes to the induction of antibody-mediated glomerulonephritis via CXCL1 and CXCL2. J Am Soc Nephrol 2007, 18(6):1732-1739.

38. Sharma K, McCue P, Dunn SR: Diabetic kidney disease in the $\mathrm{db} / \mathrm{db}$ mouse. Am J Physiol Renal Physiol 2003, 284(6):F1138-F1144.

39. Lawler J, Sunday M, Thibert V, Duquette M, George EL, Rayburn H, Hynes RO: Thrombospondin-1 is required for normal murine pulmonary homeostasis and its absence causes pneumonia. J Clin Invest 1998, 101 (5):982-992.

40. Crawford SE, Stellmach V, Murphy-Ullrich JE, Ribeiro SM, Lawler J, Hynes RO, Boivin GP, Bouck N: Thrombospondin-1 is a major activator of TGF-beta1 in vivo. Cell 1998, 93(7):1159-1170.

41. Ziyadeh FN: Mediators of diabetic renal disease: the case for tgf-Beta as the major mediator. J Am Soc Nephrol 2004, 15(Suppl 1):S55-S57.

42. Raugi GJ, Lovett DH: Thrombospondin secretion by cultured human glomerular mesangial cells. Am J Pathol 1987, 129(2):364-372.

43. Wahab NA, Schaefer L, Weston BS, Yiannikouris O, Wright A, Babelova A, Schaefer R, Mason RM: Glomerular expression of thrombospondin-1, transforming growth factor beta and connective tissue growth factor at different stages of diabetic nephropathy and their interdependent roles in mesangial response to diabetic stimuli. Diabetologia 2005, 48(12):2650-2660.

44. Adler SG, Feld S, Striker L, Striker G, LaPage J, Esposito C, Aboulhosn J, Barba L, Cha DR, Nast CC: Glomerular type IV collagen in patients with diabetic nephropathy with and without additional glomerular disease. Kidney Int 2000, 57(5):2084-2092.

45. Leehey DJ, Song RH, Alavi N, Singh AK: Decreased degradative enzymes in mesangial cells cultured in high glucose media. Diabetes 1995, 44(8): 929-935.

46. Lam S, van der Geest RN, Verhagen NA, Daha MR, van Kooten C: Secretion of collagen type IV by human renal fibroblasts is increased by high glucose via a TGF-beta-independent pathway. Nephrol Dial Transplant 2004, 19(7):1694-1701.

47. Whiteside $\mathrm{Cl}$, Dlugosz JA: Mesangial cell protein kinase $\mathrm{C}$ isozyme activation in the diabetic milieu. Am J Physiol Renal Physiol 2002, 282(6): F975-F980.

48. Kinoshita M, Nakamura T, Ihara M, Haraguchi T, Hiraoka Y, Tashiro K, Noda M: Identification of human endomucin-1 and -2 as membrane-bound Osialoglycoproteins with anti-adhesive activity. FEBS Lett 2001, 499(1-2):121-126.

49. Larsson M, Majeed M, Ernst JD, Magnusson KE, Stendahl O, Forsum U: Role of annexins in endocytosis of antigens in immature human dendritic cells. Immunology 1997, 92(4):501-511.

50. Park JE, Lee DH, Lee JA, Park SG, Kim NS, Park BC, Cho S: Annexin A3 is a potential angiogenic mediator. Biochem Biophys Res Commun 2005, 337 (4):1283-1287.

51. Pu L, Fan JM: Effects of pioglitazone on the transdifferentiation and CTGF expression of renal tubular epithelial-myofibroblast in vitro. Sichuan Da Xue Xue Bao Yi Xue Ban 2010, 41(1):15-19.

52. Ferdous Z, Wei VM, lozzo R, Hook M, Grande-Allen KJ: Decorintransforming growth factor- interaction regulates matrix organization and mechanical characteristics of three-dimensional collagen matrices. J Biol Chem 2007, 282(49):35887-35898.

53. Mohan RR, Gupta R, Mehan MK, Cowden JW, Sinha S: Decorin transfection suppresses profibrogenic genes and myofibroblast formation in human corneal fibroblasts. Exp Eye Res 2010, 91(2):238-245.

54. Williams KJ, Qiu G, Usui HK, Dunn SR, McCue P, Bottinger E, lozzo RV, Sharma K: Decorin deficiency enhances progressive nephropathy in diabetic mice. Am J Pathol 2007, 171(5):1441-1450.

doi:10.1186/1471-2369-13-70

Cite this article as: Brunskill and Potter: Changes in the gene expression programs of renal mesangial cells during diabetic nephropathy. $B M C$ Nephrology 2012 13:70.

\section{Submit your next manuscript to BioMed Central and take full advantage of:}

- Convenient online submission

- Thorough peer review

- No space constraints or color figure charges

- Immediate publication on acceptance

- Inclusion in PubMed, CAS, Scopus and Google Scholar

- Research which is freely available for redistribution 